\title{
A Vector Generalized Finite Element-Boundary Integral Formulation for Scattering from Cavity-backed Apertures
}

\author{
O. Tuncer*, B. Shanker, and L. C. Kempel \\ 2120 Engineering Building, Dept. ECE, Michigan State University, \\ East Lansing, MI 48824, E-mail:tunceroz@msu.edu
}

\section{Introduction}

Vector generalized finite element method (VGFEM) was recently developed for the solution of electromagnetic problems [2]. Theoretically, VGFEM is a powerful technique as it permits the use of a wide range of function spaces. Indeed, the method provides a rigorous framework for including approximation spaces seamlessly within a simulation. Doing so can provide better approximation, and perhaps, more efficient simulations. The method was successfully applied to canonical closed domain problems both in time and frequency domain. Since then, our goal has been to develop the accompanying suite of technologies that enable the simulation of more practical problems. This paper constitutes one such attempt, where we extend the application of the method to open domain problems by developing a hybrid VGFEM-Boundary Integral (VGFEM-BI) technique.

The formulation of VGFEM-BI compared to hybrid FEM-BI is not straightforward. Difficulties arise in implementation boundary conditions, as imposition of Dirichlet boundary conditions in VGFEM is not straightforward. The basis function space is not interpolatory. In addition, unlike FEM, auxiliary space of surface basis functions that is defined at the aperture can not be used with VGFEM as these basis functions do not satisfy the Babuska-Brezzi condition. This paper presents the means through which these difficulties can be overcome. The rest of the paper is organized as follows: In the next Section, we present a very brief overview of the the formulation. This is followed by a plethora of results that compare data obtained from VGFEM-BI against FEM-BI data and measurements. Finally, a summary of findings and directions for the future research are presented in the last Section.

\section{Theory}

Here, we formulate hybrid VGFEM-BI technique for the analysis of scattering from cavity-backed aperture in an infinite ground plane at $z=0$. The problem domain is divided into 2 regions: free space region, $\Omega_{f}$, above cavity $(z>0)$ and cavity region, $\Omega$, for $(-c<z<0)$, where $c$ is the depth of cavity. We assume that the cavity region is source free and filled with a homogenous material having a relative permittivity $\epsilon_{r}$ and a relative permeability $\mu_{r}$. Using equivalence principle and image theory, electromagnetic fields in these regions are decoupled and the fields in $\Omega$ are solved numerically by applying boundary conditions at the aperture. Electric field, $\mathbf{u}(\mathbf{r})$, in region $\Omega$, whose boundary is denoted by $\partial \Omega:=S=\bigcup_{i} S_{i}$, satisfies the vector Helmholtz equation 


$$
\begin{array}{r}
\nabla \times \frac{1}{\mu_{r}} \nabla \times \mathbf{u}(\mathbf{r})-k_{0}^{2} \epsilon_{r} \mathbf{u}(\mathbf{r})=0 \\
\oiint_{i}\{\mathbf{u}(\mathbf{r})\}=\mathbf{g}_{\mathbf{i}}(\mathbf{r}), \forall \in S_{i}
\end{array}
$$

where $k_{o}$ is the free space wavenumber, $\beta_{i}$ is a differential operator, and $\mathbf{g}_{i}(\mathbf{r})$ is a boundary condition imposed on $S_{i}$. In order to solve Eq. (1) using VGFEM, the computation domain is first sampled and overlapping partition of unity domains are defined around each node such that $\Omega \subset \bigcup_{i} \Omega_{i}$. Then, a vector basis function space $\mathbf{V}=\operatorname{span}\left\{\psi_{i}(\mathbf{r}) \nabla \times\left(\hat{c} v_{i, m}^{p}(\mathbf{r})\right), \quad \psi_{i}(\mathbf{r}) \nabla \times \nabla \times\left(\hat{c} v_{i, n}^{p}(\mathbf{r})\right)\right\}$ is defined on each $\Omega_{i}$ as a product of $\mathrm{PU}$ function $\psi_{i}(\mathbf{r})$ and local approximation function $v_{i, n}^{p}$ [2]. As the problem is solved using the VGFEM with Galerkin's method, the following boundary condition [3] is imposed at the aperture,

$$
\hat{z} \times \frac{1}{\mu_{r}}[\nabla \times \mathbf{u}(\mathbf{r})]-2 k_{0}^{2} \hat{z} \times \int_{S_{a}}[\hat{z} \times \mathbf{u}(\mathbf{r})] \cdot \overline{\bar{G}}_{0}\left(\mathbf{r}, \mathbf{r}^{\prime}\right) d S^{\prime}=-2 j k_{0} Z_{0}\left[\hat{z} \times \mathbf{H}^{i}(\mathbf{r})\right],
$$

where $\mathbf{H}^{i}(\mathbf{r})$ is the incident magnetic field, and $k_{0}$ and $Z_{0}$ are the free space wavenumber and impedance, respectively. The function $\overline{\bar{G}}_{0}$ is the free space dyadic green's function. For a smoother convergence, boundary condition on the normal components of the field

$$
\hat{z} \cdot \epsilon_{r} \mathbf{u}(\mathbf{r})-2 \hat{z} \cdot \int_{S_{a}}\left[(\mathbf{u}(\mathbf{r}) \times \hat{z}) \times \nabla G_{0}\left(\mathbf{r}, \mathbf{r}^{\prime}\right)\right] d S^{\prime}=2 \mathbf{E}^{i}(\mathbf{r}) \cdot \hat{z}
$$

is also imposed, where $\mathbf{E}^{i}(\mathbf{r})$ is the incident electric field and $G_{0}$ is the free space green's function. In addition, Nitsche's method is applied for the Dirichlet boundary condition on the PEC walls. With these boundary conditions, the bilinear form of the VGFEM-BI is written as

$$
\begin{array}{r}
\int_{\Omega_{i}}(\nabla \times \mathbf{w}) \cdot\left(\frac{1}{\mu_{r}} \nabla \times \mathbf{u}\right) d \Omega-k_{0}^{2} \epsilon_{r} \int_{\Omega_{i}} \mathbf{w} \cdot \mathbf{u} d \Omega+\beta \int_{\Omega_{i}}(\nabla \cdot \mathbf{w})(\nabla \cdot \mathbf{u}) d \Omega+ \\
\int_{S_{p e c}}\left[(\mathbf{w} \times \hat{n}) \cdot\left(\frac{1}{\mu_{r}} \nabla \times \mathbf{u}\right)+(\mathbf{u} \times \hat{n}) \cdot\left(\frac{1}{\mu_{r}} \nabla \times \mathbf{w}\right)\right] d S+ \\
\beta_{1} \int_{S_{p e c}}(\mathbf{w} \times \hat{n}) \cdot(\mathbf{u} \times \hat{n}) d S-2 k_{0}^{2} \int_{S_{a}}\left[(\mathbf{w} \times \hat{z}) \cdot \int_{S_{a}} G_{0}(\mathbf{u} \times \hat{z}) d S^{\prime}\right] d S- \\
2 \oint_{C}\left[\int_{S_{a}} G_{0} \nabla^{\prime} \cdot(\mathbf{u} \times \hat{z}) d S^{\prime}-\oint_{C} G_{0}(\mathbf{u} \times \hat{z}) \cdot \hat{n_{l}} d l^{\prime}\right](\mathbf{w} \times \hat{z}) \cdot \hat{n_{l}} d l+ \\
2 \int_{S_{a}}\left[\int_{S_{a}} G_{0} \nabla^{\prime} \cdot(\mathbf{u} \times \hat{z}) d S^{\prime}-\oint_{C} G_{0}(\mathbf{u} \times \hat{z}) \cdot \hat{n_{l}} d l^{\prime}\right] \nabla \cdot(\mathbf{w} \times \hat{z}) d S+ \\
\beta_{2} \int_{S_{a}}(\mathbf{w} \cdot \hat{z})\left(\epsilon_{r} \mathbf{u} \cdot \hat{z}\right) d S-2 \beta_{2} \int_{S_{a}}(\mathbf{w} \cdot \hat{z})\left[\hat{z} \cdot \int_{S_{a}}\left[(\mathbf{u} \times \hat{z}) \times \nabla G_{0}\left(\mathbf{r}, \mathbf{r}^{\prime}\right)\right] d S^{\prime}\right] d S= \\
2 j k_{0} Z_{0} \int_{S_{a}}(\mathbf{w} \times \hat{z}) \cdot \mathbf{H}^{i}(\mathbf{r}) d S+2 \beta_{2} \int_{S_{a}}(\mathbf{w} \cdot \hat{z})\left(\mathbf{E}^{i}(\mathbf{r}) \cdot \hat{z}\right),
\end{array}
$$

where $\mathbf{w}$ represents testing functions, $\Omega_{i}$ is the volume of the support domain of the testing function, $S_{p e c}$ and $S_{a}$ are the surfaces of the domain of testing function on the PEC and aperture respectively, $\hat{n}$ is the outward unit normal vector of the support domain, and $\hat{n}_{l}$ is the outward normal vector of the contour $C$ that bounds the 
partition of unity domain. The constants $\beta, \beta_{1}$ and $\beta_{2}$ contribute uniform convergence [2]. Unlike FEM formulation, the line integrals appear in the formulation as the derivatives of the basis functions may not be continuous across the boundaries. In FEM-BI, auxiliary basis functions are defined at the aperture. However, it is well known that the definition of this auxiliary space is not possible in VGFEM as it does not satisfy the Babuska-Brezzi condition. Thus, volumetric vector basis functions are forced to satisfy boundary conditions. This is achieved by constructing the PU domains from the geometry brick elements such that there exists boundary PU domains that are defined around each boundary node. Note, the singular integrals in Eq. (4) are evaluated using rules presented in [4], and the other integrals are evaluated using Gauss-Legendre rule for the prescribed accuracy.

\section{Results}

We validate the VGFEM-BI by simulating scattering from cavity backed apertures. First, RCS of an empty cavity with the size of $1.5 \lambda_{0} \times 1.5 \lambda_{0} \times 0.6 \lambda_{0}$ is simulated. Legendre polynomials is used on a cubic PU domain with an approximate edge length of $h_{p}=\lambda_{0} / 2.5$. Fig. 1(a) compares RCS of the cavity computed by VGFEM-BI and FEM-BI [5] and shows the - $p$ convergence of VGFEM-BI. VGFEM-BI results with higher orders match with FEM-BI results well. Next, we simulate RCS of a cavity

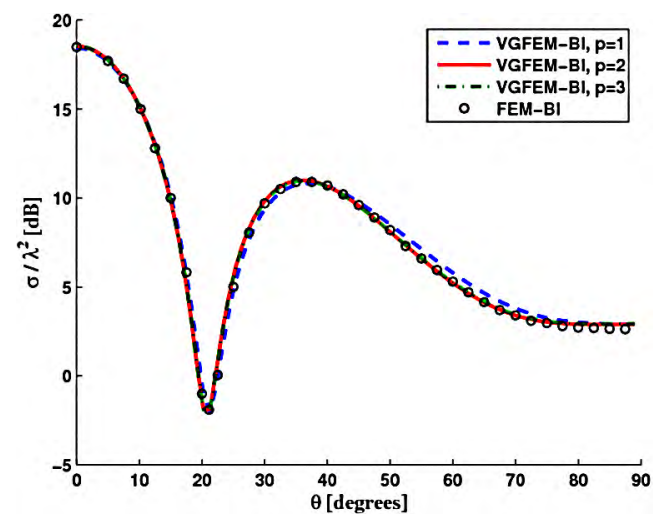

(a) $1.5 \lambda_{0} \times 1.5 \lambda_{0} \times 0.6 \lambda_{0}$ empty cavity

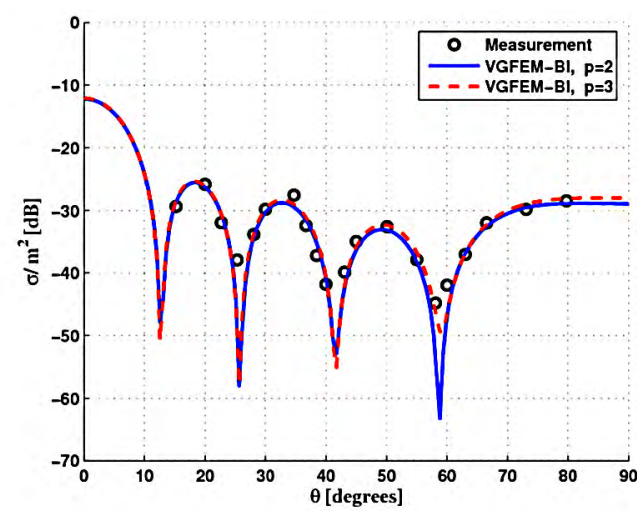

(b) 2.89 in $\times 2.1$ in $\times 0.057$ in filled cavity.

Figure 1: Backscattered RCS of an empty and filled cavities for $E^{i}=\hat{\theta} e^{-j \mathbf{k} . \mathbf{r}}$. RCS of filled cavity with $\epsilon_{r}=4$ is compared against measurement [6] at $9.2 \mathrm{GHz}$.

with the size of 2.89 in $\times 2.1$ in $\times 0.057$ in at $9.2 \mathrm{GHz}$. The cavity is filled with a material having $\epsilon_{r}=4$. VGFEM-BI results are very close to the measurement results obtained in [6] as shown in Fig. 1(b). Finally, RCS of a $1 \lambda_{0} \times 0.25 \lambda_{0} \times 0.25 \lambda_{0}$ cavity filed with a material having $\epsilon_{r}=7-j 1.5$ and $\mu_{r}=1.8-j 0.1$ is simulated. Cubic PU domain with edge length of approximately $h_{p}=\lambda_{0} / 6$ and the polynomials order of $p=2$ are used for VGFEM-BI. Figure 2 shows excellent agreement between VGFEM-BI and FEM-MoM results [7].

\section{Conclusion}

In this paper, we have developed a hybrid VGFEM-BI formulation for the analysis of scattering from filled cavities. Since surface basis functions are not supported in 


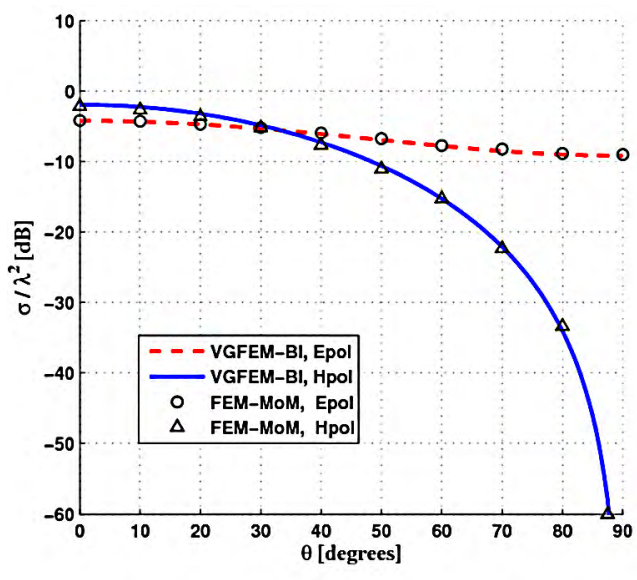

(a) $\phi_{\text {inc }}=\pi / 2$

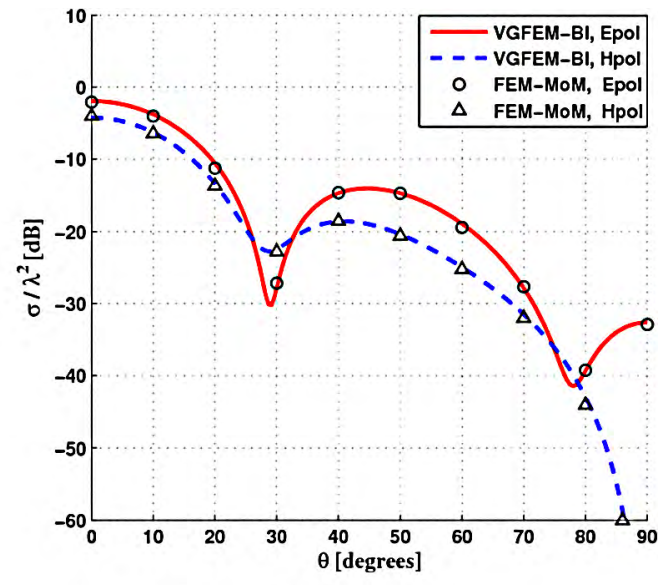

(b) $\phi_{\text {inc }}=0$

Figure 2: Backscattered RCS of $1 \lambda_{0} \times 0.25 \lambda_{0} \times 0.25 \lambda_{0}$ cavity filled with $\epsilon_{r}=7-j 1.5$ and $\mu_{r}=1.8-j 0.1$

the framework of VGFEM, volumetric vector basis functions are forced to impose boundary conditions at the aperture. This is achieved by constructing conformal PU domains from the geometry mesh. The method has been validated by comparing the simulated radar cross sections of filled cavities against measurement and FEM-BI data. Our current research is on the application of the method for large scattering and radiation problems using tailored basis functions, and these results will be presented at the conference.

\section{References}

[1] C. Lu and B. Shanker, "Hybrid Boundary Integral-Generalized (Partition of Unity) Finite-Element Solvers for the Scalar Helmholtz Equation," IEEE Trans. on Magnetics 43, 3, 1002-1012, 2007.

[2] C. Lu and B. Shanker, "Generalized Finite Element Method for Vector Electromagnetic Problems ," IEEE Trans. on Ant. and Prop., 55, 5, 1369-1381, 2007.

[3] Jianming Jin, The Finite Element Method in Electromagnetics, New York, Wiley.

[4] M. A. Khayat and D. R. Wilton, "Numerical Evaluation of Singular and NearSingular Potential Integrals," IEEE Trans. on Ant. and Prop., 53, 10, 3180-3190, 2005 .

[5] J. M. Jin and L. Volakis, "A Special Higher Order Finite-Element Method for Scatttering by Deep Cavities," IEEE Trans. on Ant. and Prop., 48, 5, 609-703, 2000.

[6] J. M. Jin and J. L. Volakis, "A Hybrid Finite Element Method for Scattering and Radiation by Microstrip Patch Antennas and Arrays Residing in a Cavity," IEEE Trans. on Ant. and Prop., 39, 11, 1598-1604, 1991.

[7] C. J. Reddy, M. D. Deshpande, C .R Cockrell and F. B Beck, "Electromagnetic Scattering Analysis of a Three-Dimensional-Cavity-Backed Aperture in an Infinite Ground Plane Using a Combined Finite Element Method/Method of Moments Approach," NASA Technical Paper 3544, 1995. 